\title{
Impact of inpatient rehabilitation services on the functional levels of cancer patients at King Fahad Medical City, Riyadh, Saudi Arabia
}

Amani AlJohi, MSc, Sherwynn Javison, MSc, Suzan Lubbad, BSc, Yara Bashawri, MPH, Abdullah Bany Hamdan, MSc.

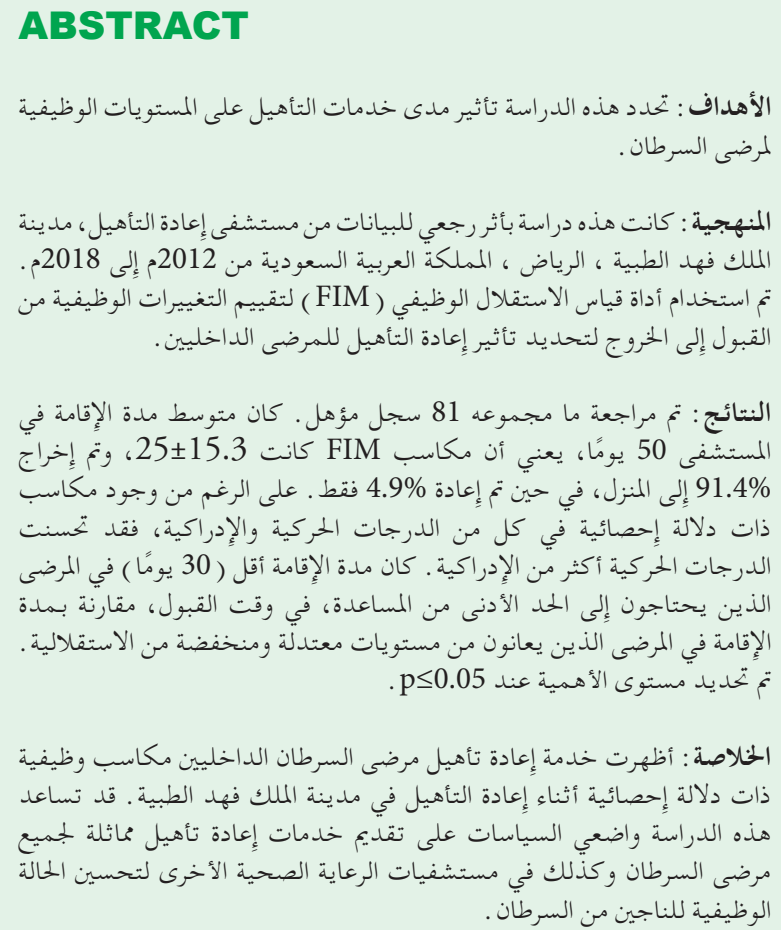

Objectives: To identify the impact of inpatient rehabilitation services on the functional levels of cancer patients.

Methods: This was a retrospective study of data from the Rehabilitation Hospital, King Fahad Medical City, Riyadh, Saudi Arabia, from 2012 to 2018. The functional independence measure (FIM) tool was used to assess functional changes from admission to discharge to determine the impact of inpatient rehabilitation.

Results: A total of 81 eligible records were reviewed. The median hospital length of stay (LOS) was 50 days, mean \pm SD of the FIM gain was $25(15.3)$, and $91.4 \%$ were discharged home, while only $4.9 \%$ were readmitted. Although statistically significant gains were observed in both motor and cognitive scores, motor scores improved more than the cognitive. The LOS was less (30 days) in patients requiring minimal assistance, at the time of admission, compared to the LOS in patients with moderate and low levels of independence. The level of significance was set at $p \leq 0.05$

Conclusions: In patient cancer rehabilitation service demonstrated statistically significant functional gains during rehabilitation at King Fahad Medical City. This study may help the policymakers to provide similar rehabilitation services to all cancer patients as well as in other health care hospitals to improve the functional status of cancer survivors.

Keywords: cancer survivors, FIM scale, inpatient rehabilitation

Saudi Med J 2020; Vol. 41 (9): 984-989

doi: 10.15537/smj.2020.9.25309

From the Physical Therapy Department (AlJohi), Rehabilitation Hospital, King Fahad Medical City; from the Comprehensive Cancer Center (Javison, Bany Hamdan); from the Physical Therapy Department, Rehabilitation Hospital (Lubbad); from the Biostatistical Department, Research Center (Bashawri), King Fahad Medical City, Riyadh, Kingdom of Saudi Arabia.

Received 31st May 2020. Accepted 29th July 2020

Address correspondence and reprint request to: Amani AlJohi, Department of Physical Therapy, Rehabilitation Hospital, King Fahad Medical City, Riyadh, Kingdom of Saudi Arabia. E-mail:a_aljohi@yahoo.com ORCID ID: https://orcid.org/0000-0001-5619-3559

$T$ he majority of cancer patients have some degree of physical impairment. Approximately one-third of them are limited in their basic activities of daily living (ADL) and have difficulty walking because of disease progression or treatment. ${ }^{1-3}$ The physical and psychological impairment of cancer survivors negatively affects their quality of life (QOL). 4.5 In 2018, the Global Burden of Disease Cancer Collaboration 
reported that there were 17.2 million people living with cancer worldwide between 2006 and 2016, and the incidence of cancer has increased by approximately $28 \% .^{6}$ Fortunately, the number of cancer survivors continues to increase, primarily as a result of advances in cancer treatment and early diagnoses. While this is positive news, it comes with a heavy burden of disability for survivors who account for a total of 213.2 million disability-adjusted life years. ${ }^{6}$ Therefore, this high disability rate has increased the demand for early physical rehabilitation.

The importance of rehabilitation referrals among cancer patients has already been recognized, since they are an integral part of care coordination among healthcare providers. Inpatient rehabilitation services play a significant role in overall QOL improvement and affect many patients' ability to cope with their disabilities. ${ }^{7,8}$ Previous studies have reported that cancer patients benefit from inpatient rehabilitations. ${ }^{1-4}$ However, functional impairments related to cancer are often not referred for specialized rehabilitation services as the main focus of oncologists remains the treatment of the disease rather than the functional deficits it causes. In addition, specialists in rehabilitation medicine and rehabilitation facilities where they practice are keenly aware of the burden and escalating costs that accompany the special care requirements of cancer patientss. ${ }^{6}$

Functional improvement achieved through inpatient rehabilitation is associated with prolonged survivorship among cancer patients. ${ }^{10}$ The functional independence measure (FIM) is widely used in the context of inpatient cancer rehabilitation. This is a valid and reliable tool to assess the level of independence in basic ADL. It has been found to be a reliable tool not only for cancer survivors but also in stroke and other neurological disorders and has been widely used in many countries, including Europe, the UK, and Australias. ${ }^{11-15}$

However, cancer patients with functional impairment are rarely referred for rehabilitation services. In Saudi Arabia, there were 16,210 cancer cases in 2015, and the number has increased yearly. ${ }^{16}$ The major barriers to optimizing the delivery of oncology rehabilitation are the lack of proper identification of patients' functional impairment and gaps in multidisciplinary communication. Moreover, oncologists and hematologists are unfamiliar with the

Disclosure. Authors have no conflict of interests, and the work was not supported or funded by any drug company. concept of rehabilitation has led to reduced referrals. ${ }^{17}$ Furthermore, the complexity of cancer patient conditions and the lack of healthcare providers who specialize in cancer rehabilitation may negatively impact their acceptance of cancer patients as inpatients in their facilities. 'Providing proper attention and services to cancer patients is undoubtedly a significant challenge nationwide when facilities are limited in terms of inpatient rehabilitation. Indeed, there have been no studies regarding the influence of inpatient rehabilitation services on cancer patients in Saudi Arabia. Therefore, the purpose of this study was to identify the impact of inpatient rehabilitation services on the functional levels of cancer patients at a tertiary health care hospital, with the hopes of facilitating opportunities for more cancer patients and survivors to receive similar services in the future.

Methods. The study used a retrospective chart review of related data gathered between January 2012 and December 2018. It was conducted at the Rehabilitation Hospital, King Fahad Medical City, Riyadh, Saudi Arabia. The King Fahad Medical City-Rehabilitation Hospital (KFMC-RH) is one of the main and largest Ministry of Health tertiary inpatient rehabilitation facility (IRF) and provides services to referred patients in Riyadh free of cost. This facility provides a holistic rehabilitation approach by an interdisciplinary team led by a physiatrist. Based on the patients' activity limitation, all patients receive multidisciplinary management including nursing, physical therapy, occupational therapy, speech or swallowing, and other services. In addition, patients receive support services such as art therapy, social support, and recreation therapy.

All adult cancer patients ( $\geq 14$ years old) who were admitted for rehabilitation at this facility from the year 2012 to 2018 were included. Patients' prognosis more than 6 months and were not receiving any chemo or radiotherapy. Records were excluded if there were missing FIM scores, if cancer was a secondary diagnosis, any metastasis, and when the patient had already received rehabilitation services in other facilities or at home. This study was approved by the Institutional Review Board of King Fahad Medical City. The patients' level of functional independence at the time of admission and at discharge was noted.

Social demographic information, comorbidity, and length of stay were collected from patient records. The FIM tool is widely used to identify physical and cognitive limitations that indicate the level of independence, and to assess functional gain during inpatient cancer rehabilitation. The FIM consists of 
18 items and is subdivided to a motor and cognitive components. Motor component is assessed on 4 domains of function: i) self-care (6 items) ii) sphincter control (2 items), iii) mobility/transfers (3 items), and iv) locomotion (2 items). Communication (2 items), and social cognition (3 items) are cognitive function domains. All the items are scored on a 7 point ordinal scale; 1- total assistance and 7-complete independence. This functional measure has a total score range from 18-126, a greater score indicates a higher level of independence. ${ }^{15}$

Statistical analysis. Demographic and clinical characteristics of the study patiens were reported as mean (SD), median (25th percentile, 75 th percentile) or counts (percentage, as appropriate. Differences between admission and discharge FIM scores were compared using Wilcoxon signed-rank test. All statistical analyses were conducted using Statistical Package for Social Sciences version 25.0 (SPSS Inc., Chicago, IL, USA). A 2-tailed test with a $p$-value of 0.05 was considered significant.

Results. A total of 86 medical records of cancer patients admitted for inpatient rehabilitation at King Fahad Medical City were included. Five records were excluded because of inadequate data, leaving the total sample at 81 . The mean age was 47.38 years (SD 17.18)

Table 1 - Summary of patient demographics and medical characteristics.

\begin{tabular}{lc}
\hline Characteristics & $\mathbf{n}(\%)$ \\
\hline Age (years) (mean \pm SD) & $47.38(17.18)$ \\
Gender & $37(45.7)$ \\
Males & $44(54.3)$ \\
Females & \\
Referral source & $38(46.9)$ \\
Comprehensive Cancer Center & $43(53.1)$ \\
Neurosurgery Ward & \\
Diagnosis & $5(6.2)$ \\
Breast cancer & $6(7.4)$ \\
Multiple myeloma & $14(17.3)$ \\
Lymphoma & $47(58.0)$ \\
Brain tumor & $9(11.1)$ \\
Others (endometrial, leukemia) & \\
Discharge & $74(91.4)$ \\
Home & $4(4.9)$ \\
Readmission to acute inpatient facility & $3(3.7)$ \\
Transfer to long-term facility &
\end{tabular}

and $54.3 \%$ of the patients were female. Out of 81 files, $38(46.9 \%)$ were retrieved from the Comprehensive Cancer Center (CCC), while 43 (53.1\%) were brain tumor cases from the neurosurgery ward. Most of the cases $(n=20 ; 24.4 \%)$ from CCC were hematologic malignancies, and 18 (22\%) were other solid tumors. The majority of participants (58\%) were diagnosed with brain tumors. The participants' characteristics are shown in Table 1.

At admission. At the time of admission, $53.1 \%$ of patients required moderate assistance, while only $37 \%$ required maximal assistance for their activities. The mean of the total FIM scores at the time of admission was 63.52 (SD 21.61), mean of the total motor scores was 3.25 (SD 15.80), and mean of the total cognitive scores 28.27 (SD 9.51).

At discharge. At the time of discharge, only $29.6 \%$ of patients required moderate assistance, and only $12.3 \%$ required maximal assistance, as measured by the FIM. The mean total FIM score was 89.35 (SD 25.59), mean FIM motor score 59.26 (SD 20.87), and mean cognitive score 30.09 (SD 8.13). There was a significant improvement in FIM scores from admission to discharge (Table 2; Figure 1). The median scores at 25th and 75th percentiles are also presented in table 2 .

Hospital length of stay (LOS). The median patient length of stay (LOS) was 50 days and ranged from 4 to 333 days. Patients requiring minimal assistance had a median LOS of 26.50 days, and their FIM efficiency was $0.69(0.48)$. All patients with a high level of independence were discharged home. Patients with a moderate level of independence had a median LOS of 43 days, and FIM efficiency was 0.69 (0.44). The majority of patients $(93 \%)$ were discharged home, while $7 \%$ were readmitted to an acute facility. Patients with a

Table 2 - Comparison of functional independence measure (FIM) scores from admission to discharge.

\begin{tabular}{lccc}
\hline FIM scores & Admission & Discharge & $P$-value \\
\hline Total FIM scores & & & \\
Mean \pm SD & $63.52 \pm 21.61$ & $89.35 \pm 25.59$ & \\
Median $\left(25^{\text {th }}, 75^{\text {th }}\right)$ & $65(49,80)$ & $96(73,110)$ & $<0.0001$ \\
FIM motor score & & & \\
Mean \pm SD & $35.25 \pm 15.80$ & $59.26 \pm 20.87$ & \\
Median $\left(25^{\text {th }}, 75^{\text {th }}\right)$ & $34(20,48)$ & $62(41,77)$ & $<0.0001$ \\
FIM cognitive score & & & \\
Mean \pm SD & $28.27 \pm 9.51$ & $30.09 \pm 8.13$ & \\
Median $\left(25^{\text {th }}, 75^{\text {th }}\right)$ & $35(23,35)$ & $35(29,35)$ & $<0.0001$ \\
\hline
\end{tabular}




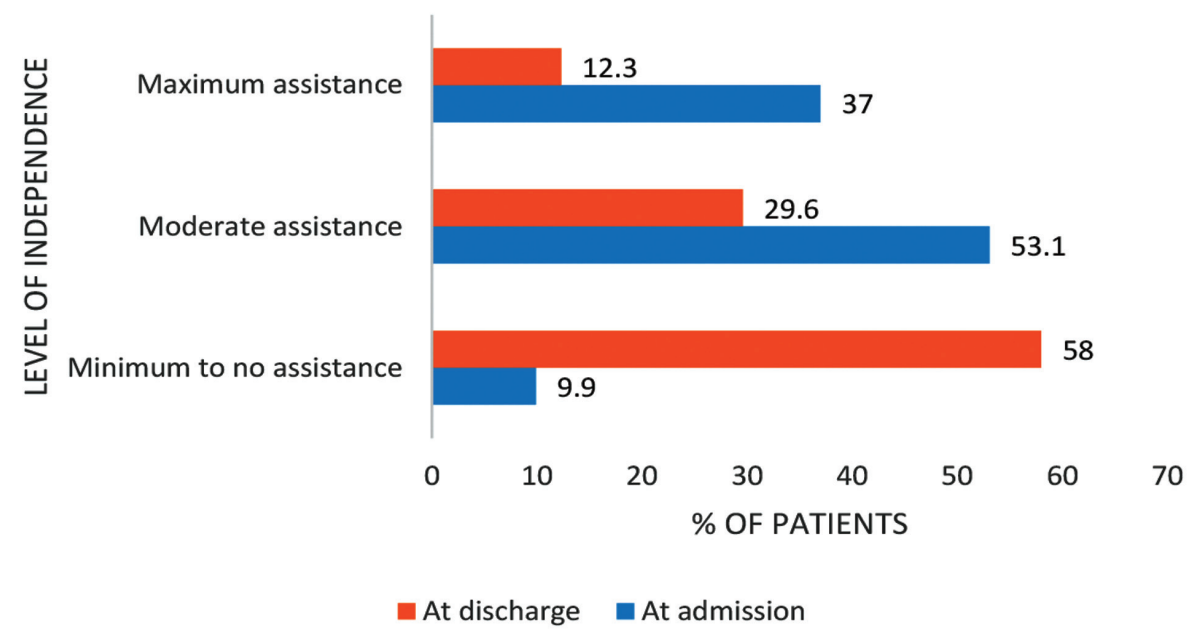

Figure 1 - Level of independence from admission to discharge.

Table 3 - Participants' level of independence, hospital length of stay, and FIM efficiency at discharge.

\begin{tabular}{|c|c|c|c|}
\hline FIM Scores & $\begin{array}{c}\text { Minimum } \\
\text { assistance }(n=8)\end{array}$ & $\begin{array}{c}\text { Moderate } \\
\text { assistance }(n=43)\end{array}$ & $\begin{array}{c}\text { Maximum } \\
\text { assistance }(n=30)\end{array}$ \\
\hline \multicolumn{4}{|l|}{ FIM admission } \\
\hline Mean \pm SD & $98.13 \pm 6.6$ & $73.16 \pm 8.76$ & $40.47 \pm 12.2$ \\
\hline Median & 97.5 & 72 & 42.5 \\
\hline $25^{\text {th }}$ & 92.5 & 65 & 29 \\
\hline $75^{\text {th }}$ & 102.5 & 82 & 50 \\
\hline \multicolumn{4}{|l|}{ FIM discharge } \\
\hline Mean \pm SD & $114.38 \pm 7.56$ & $100.19 \pm 15.17$ & $67.13 \pm 24.55$ \\
\hline Median & 117.5 & 104 & 68.5 \\
\hline $25^{\text {th }}$ & 108.5 & 94 & 48 \\
\hline $75^{\text {th }}$ & 120.5 & 112 & 84 \\
\hline$P$-value & $0.012^{*}$ & $<0.0001^{*}$ & $<0.0001^{*}$ \\
\hline \multicolumn{4}{|l|}{ FIM efficiency } \\
\hline Mean \pm SD & $0.69 \pm 0.48$ & $0.69 \pm 0.44$ & $0.48 \pm 0.41$ \\
\hline Median & 0.64 & 0.57 & 0.34 \\
\hline $25^{\text {th }}$ & 0.24 & 0.33 & 0.16 \\
\hline $75^{\text {th }}$ & 1.13 & 1.03 & 0.69 \\
\hline \multicolumn{4}{|l|}{ Length of stay (days) } \\
\hline Mean \pm SD & $29.88 \pm 14.86$ & $52.49 \pm 36.63$ & $73.93 \pm 53.81$ \\
\hline Median & 26.50 & 43 & 62.50 \\
\hline $25^{\text {th }}$ & 21.50 & 30 & 51 \\
\hline $75^{\text {th }}$ & 40.50 & 62 & 83 \\
\hline \multicolumn{4}{|l|}{ Discharge location } \\
\hline Home n (\%) & $8(100)$ & $40(93)$ & $26(86.7)$ \\
\hline Readmission n (\%) & $0 \quad(0)$ & $3(7)$ & $1 \quad(3.3)$ \\
\hline Other facilities n (\%) & $0 \quad(0)$ & $0 \quad(0)$ & $3(10.0)$ \\
\hline
\end{tabular}


low level of independence had a median LOS of 62.50 days, and their FIM efficiency was 0.48 (0.41). There was a significant difference in their FIM scores from admission to discharge, and $86.7 \%$ of patients were discharged home, $3.3 \%$ were readmitted, and $10 \%$ were transferred to long-term care facilities (Table 3).

Discussion. This study analyzed the functional efficiency of patients who received inpatient rehabilitation at KFMC over a period of 7 years. Our retrospective study found that inpatient cancer rehabilitation patients showed significant functional gain from admission to discharge, and the majority (91\%) were discharged home.

In our sample, brain tumors accounted for about $58 \%$ of inpatient cancer rehabilitation admissions. Similar results were observed in a study done in a US rehabilitation facility, and they also found that cancer survivors accounted for only $2.4 \%$ of all inpatient rehabilitation admissions between the years 2010 and 2012. ${ }^{18}$ The median LOS in our sample was 50 days, with a minimum of 4 and a maximum of 333 days. We also observed that the mean FIM gain was 25.8 (15.3) points and total FIM efficiency 0.61 (0.44), and $91.4 \%$ were discharged home. In contrast to our findings, FIM efficiency was 2 in a recent 13-year study. ${ }^{4}$ Another study by Forrest and Deike reported that LOS was 18.7 days for the cancer patients, and the FIM efficiency score was 1.12 and 0.80 in patients with primary brain tumors and those with metastasis to the brain, respectively. ${ }^{3}$ The LOS of cancer patients in our sample was relatively longer compared to the LOS, 14 days, observed in other studies. ${ }^{4}$ The possible reason for the observed longer LOS may be due to the presence of an outlier of 333 days, which would have affected the mean LOS in our sample. Furthermore, this may have also resulted in delayed FIM efficiency in our study.

From our findings, it was evident that patients experienced an improvement in their activities as demonstrated by their total FIM scores. Further analysis of our results showed that both motor and cognitive FIM scores improved significantly at discharge, motor gain 24 (14) more so than the cognitive gain 1.8 (3.2). The impact of rehabilitation was therefore greater on physical function than cognitive ability. The existing literature also showed that improvements in FIM scores resulted in decreased strain on resources and burden of caregivers. ${ }^{3,4}$

Common barriers to cancer rehabilitation were lack of physician/oncologist knowledge about rehabilitation benefits, lack of rehabilitation facilities, and lack of cooperation between interdisciplinary team members, and these factors negatively affect the functional recovery of cancer survivors. ${ }^{17}$ Apart from the above-mentioned factors, the clinical profile of cancer patients admitted to rehabilitation facilities may also affect the overall prognosis and duration of hospital stay. Aljohi et $\mathrm{al}^{17}$ reported that barriers to admitting patients with cancer are essentially the perceived chances of readmission to an acute setting. ${ }^{17}$ Our study reported that only $4.9 \%$ were readmitted to an acute care setting. In contrast to our study results, previous studies showed that approximately $18 \%$ of cancer patients with low functional capacity return to an acute setting. ${ }^{3,4,19}$ They also found that FIM scores were associated with the return rate to an acute setting. However, the other factors associated with the return of cancer patients to acute care settings are still unclear. Ours was a retrospective study from one health care setting involving a small number of cancer patients and hence lacks sufficient information about the reason why cancer patients were admitted back to an acute setting. Hence, further studies to identify the factors associated with readmission to acute care are warranted.

Inpatient cancer rehabilitation provides post-acute care including medical management, physiotherapy, and occupational and speech therapy, and this helps to restore functional ability that allows cancer survivors to be independent in their daily activities. It also helps to manage impairments related to cancer treatment. ${ }^{20}$ Similar to previous research, ${ }^{21}$ our study results also found that inpatient rehabilitation helps to improve the functional status of cancer patients. Often, the impairments in cancer survivors were unnoticed or untreated, which leads to physical disability and further leads to distress and reduced QOL. Research has shown that rehabilitation helps to reduce physical disability and improve overall QOL of cancer survivors. ${ }^{22}$

Although our study is the first of its kind to assess the impact of inpatient rehabilitation on functional recovery, it is worth mentioning a few limitations. With a retrospective design and small sample size, it was not possible to explain the factors related to functional recovery or the reasons for readmission to acute care. It is also not possible to know the functional status of patients after discharge or how well the patients who were not admitted to inpatient rehabilitation performed.

In conclusions, patients with cancer receiving inpatient rehabilitation have significantly improved functional capacity from admission to discharge. One of 
the challenges of cancer rehabilitation is that oncologists and other acute care physicians are unaware of the benefits of inpatient rehabilitation. Therefore, further studies to examine the factors that lead to reduced referrals are warranted. The present study found that cancer survivors who referred to rehabilitation improved their function, and therefore, inpatient rehabilitation should be considered for all cancer patients with activity limitations.

Acknowledgment. We thank Rehab Hospital and Research Centre at King Fahad Medical City, Riyadh, Saudi Arabia for their support in successful completion of this study. We would like to thank Wiley editing services (www.wileyeditingservices.com) for English language editing.

\section{References}

1. Ugolini D, Neri M, Cesario A, Bonassi S, Milazzo D, Bennati $\mathrm{L}$, et al. Scientific production in cancer rehabilitation grows higher: a bibliometric analysis. Support Care Cancer 2012; 20: 1629-1638.

2. Cheville AL, Troxel AB, Basford JR, Kornblith AB. Prevalence and treatment patterns of physical impairments in patients with metastatic breast cancer. J Clin Oncol 2008; 26: 2621.

3. Forrest G, Deike D. The impact of inpatient rehabilitation on outcome for patients with cancer. J Community Support Oncology 2018; 16: e138-e144.

4. Gallegos-Kearin V, Knowlton SE, Goldstein R, Mix J, Zafonte R, Kwan M, et al. Outcome Trends of Adult Cancer Patients Receiving Inpatient Rehabilitation: A 13-Year Review. Am J Phys Med Rehabil 2018; 97: 514-522.

5. Ng AH, Gupta E, Fontillas RC, Bansal S, Williams JL, Park M, et al. Patient-reported usefulness of acute cancer rehabilitation. PM R 2017; 9: 1135-1143.

6. Fitzmaurice C, Akinyemiju TF, Al Lami FH, Alam T, AlizadehNavaei R, Allen C, et al. Global, regional, and national cancer incidence, mortality, years of life lost, years lived with disability, and disability-adjusted life-years for 29 cancer groups, 1990 to 2016: a systematic analysis for the global burden of disease study. JAMA Oncology 2018; 4: 1553-1568.

7. Riedl D, Giesinger JM, Wintner LM, Loth FL, Rumpold G, Greil R, et al. Improvement of quality of life and psychological distress after inpatient cancer rehabilitation. Wien Klin Wochenschr 2017; 129: 692-701.

8. Horsboel TA, Bultmann U, Nielsen CV, Nielsen B, Andersen NT, de Thurah A. Are fatigue, depression and anxiety associated with labour market participation among patients diagnosed with haematological malignancies? A prospective study. Psychooncology 2015; 24: 408-415.
9. Cheville AL, Kornblith AB, Basford JR. An examination of the causes for the underutilization of rehabilitation services among people with advanced cancer. Am J Phys Med Rehabil 2011; 90: S27-S37.

10. Saotome T, Klein L, Faux S. Cancer rehabilitation: a barometer for survival? Support Care Cancer 2015; 23: 3033-3041.

11. Miki E, Yamane S, Yamaoka M, Fujii H, Ueno H, Kawahara $\mathrm{T}$ et al. Validity and reliability of the Japanese version of the FIM + FAM in patients with cerebrovascular accident. Scand J Occup Ther 2015; 13: 1-7.

12. Jorge LL M F, Hara AC, Battistella LR. Brazilian version of the Functional Assessment Measure: cross-cultural adaptation and reliability evaluation. Int J Rehabil Res 2011; 34: 89-91.

13. Hadian MR, Yekaninejad MS, Salehin F, Razavi SH, Javidan AN, Pakpour AH et al. Cross-cultural adaptation and reliability evaluation of Iranian version of Functional Assessment Measure in spinal cord injury patients. Neurol Neurochir Pol 2012; 46: 351-356.

14. Granger CV, Deutsch A, Russell C, Black T, Ottenbacher KJ. Modifications of the FIM instrument under the inpatient rehabilitation facility prospective payment system. Am J Phys Med Rehabil 2007; 86: 883-892.

15. Granger CV, Markello SJ, Graham JE, Deutsch A, Reistetter TA, Ottenbacher KJ. The uniform data system for medical rehabilitation: report of patients with traumatic brain injury discharged from rehabilitation programs in 2000-2007. Am J Phys Med Rehabil 2010; 89: 265-278.

16. Saudi Health Council, National Health Information Centre Saudi Cancer Registry. Cancer Incidence Report, Saudi Arabia. [cited 2015]. Available from: https://nhic.gov.sa/eServices/ Documents/E\%20SCR\%20final\%206\%20NOV.pdf

17. AlJohi AA, AlKathiri FM, Ajaj KF, Buragadda S, Madi SM. Barriers to cancer rehabilitation: healthcare professionals' perspective in Saudi Arabia. Physikalische Medizin, Rehabilitationsmedizin, Kurortmedizin 2019; 58: 317-322.

18. Fu JB, Morishita S, Yadav R. Changing Paradigms in the Rehabilitation of Inpatients with Brain Tumors. Curr Phys Med Rehabil Rep 2018; 2:115-120.

19 Asher A, Roberts PS, Bresee C, Zabel G, Riggs RV, Rogatko A. Transferring inpatient rehabilitation facility cancer patients back to acute care (TRIPBAC). PM R 2014; 6: 808-813.

20. Silver JK, Baima J, Mayer RS. Impairment-driven cancer rehabilitation: an essential component of quality care and survivorship. CA Cancer J Clin 2013; 63: 295-317.

21. Mix JM, Granger CV, LaMonte MJ, Niewczyk P, DiVita MA, Goldstein R, et al. Characterization of cancer patients in inpatient rehabilitation facilities: A retrospective cohort study. Arch Phys Med Rehabil 2017; 98: 971-980.

22. Rath HM, Ullrich A, Otto U, Kerschgens C, Raida M, HagenAukamp C, et al. Psychosocial and physical outcomes of in- and outpatient rehabilitation in prostate cancer patients treated with radical prostatectomy. Support Care Cancer 2016; 24 : 2717-2726. 